\title{
Preimplant Hearing Aid Fittings and Aided Audibility for Pediatric Cochlear Implant Recipients
}

DOI: $10.3766 /$ jaaa. 17126

\author{
Alissa Nickerson* \\ Lisa S. Davidson $\dagger \ddagger \S$ \\ Rosalie M. Uchanski†
}

\begin{abstract}
Background: Audibility of speech for children with hearing loss $(\mathrm{HL})$ depends on the degree of $\mathrm{HL}$ and the fitting of the hearing aids (HAs) themselves. Many studies on cochlear implant (CI) users have demonstrated that preimplant hearing is associated with postimplant outcomes, but there have been very few reports on the fitting of HAs before surgery.
\end{abstract}

Purpose: The aims of this study were to characterize HA fittings and aided audibility of speech for pediatric HA users with severe to profound $\mathrm{HL}$ and to examine the relation between preimplant aided audibility and postimplant speech perception.

Research Design: A descriptive/observational and correlational study. Audiologic records of pediatric $\mathrm{Cl}$ participants involved in a larger study examining the effects of early acoustic hearing were analyzed retrospectively; when available, these records included HA verification and speech recognition performance.

Study Sample: The $\mathrm{Cl}$ participants were enrolled in audiology centers and oral schools for the deaf across the United States.

Data Collection and Analysis: To determine whether deviations from prescribed DSL target were significantly greater than zero, $95 \%$ confidence intervals of the mean deviation were calculated for each frequency $(250,500,1000,2000$, and $4000 \mathrm{~Hz})$. Correlational analyses were used to examine the relationship between preimplant aided Speech Intelligibility Indices (SIIs) and postimplant speech perception in noise. Correlational analyses were also used to explore the relationship between preimplant aided SIls and demographic data. T-tests were used to compare preimplant-aided SIls of HAs of listeners who later became users of either sequential Cls, simultaneous $\mathrm{Cls}$, or bimodal devices.

Results: Preimplant fittings of HAs were generally very close to prescriptive targets, except at $4000 \mathrm{~Hz}$ for those HAs with active frequency-lowering processing, and preimplant Slls, albeit low, were correlated with postimplant speech recognition performance in noise. These results suggest that aided audibility should be maximized throughout the HA trial for later speech recognition purposes.

Conclusions: It is recommended that HA fittings be optimized to support speech audibility even when considering implantation. In addition to the age at which HA use begins, the aided audibility itself is important in determining $\mathrm{Cl}$ candidacy and decisions regarding bimodal $\mathrm{HA}$ use.

Key Words: cochlear implants, hearing aids, hearing loss, pediatric, SII, verification

Abbreviations: $\mathrm{Cl}=$ cochlear implant(s); $\mathrm{HA}=$ hearing aid(s); $\mathrm{HL}=$ hearing loss; $\mathrm{DSL}=$ Desired Sensation Level; IQR = interquartile range; LL = lower limits; LNT = Lexical Neighborhood Test; PTA = pure-tone average; SD = standard deviation; SII = Speech Intelligibility Index; SPL = sound pressure level; UL = upper limits; UWO-PedAMP = University of Western Ontario Pediatric Audiological Monitoring Protocol

* Department of Speech and Hearing Science, University of Illinois, Champaign, IL; †Program in Audiology and Communication Science, St. Louis, MO; ¥Department of Otolaryngology, Washington University School of Medicine, St. Louis, MO; §Central Institute for the Deaf, St. Louis, M0

Corresponding author: Lisa S. Davidson, Washington University School of Medicine, St. Louis, M0 63110; Email: davidsonls@wustl.edu

NIH T35 DC008765 (Washington University Program in Audiology and Communication Sciences) and NIH R01 DC012778 (PI: LSD).

Parts of this work were presented at the 43rd Annual meeting of the American Auditory Society (AAS) in Scottsdale, AZ, March 3-5, 2016 and at the International Hearing Aid Research Conference (IHCON) in Tahoe City, CA, August 10-14, 2016. 


\section{INTRODUCTION}

$\mathrm{I}$ $\mathrm{t}$ is well known that children with hearing loss (HL) demonstrate deficits in speech and language skills compared with similarly aged children with normal hearing sensitivity (Nicholas, 2000; Geers et al, 2009; Pittman and Schuett, 2013; Davidson et al, 2014; Tomblin et al, 2014). The major reason for these deficits is the reduced ability to hear spoken language (Tomblin et al, 2014). That is, infants and children with HL have reduced audibility of speech and sounds in their environment compared with those without HL and, hence, much of spoken language is inaudible. When audibility is reduced, the ability to learn a spoken language is affected. For children with HL, hearing aids (HA) are often used as a (re)habilitation approach to improve the audibility of speech and sounds. For infants and children who do not adequately benefit from appropriately fit amplification, additional approaches, such as cochlear implant(s) (CI), may be considered.

Even though many children with severe to profound HL will eventually receive CI(s) (Sininger et al, 2010), a trial with well-fit HAs is typically recommended before CI surgery (often as part of the CI candidacy process). Before implant surgery, the everyday audibility of speech for these children will depend on both the child's degree of HL (e.g., unaided pure-tone average [PTA]) and on how well his/her HA(s) is/are fit. Many studies of pediatric CI users focus on the first item and ignore the second; they report the amount of preimplant hearing and its relation to various eventual outcomes, such as speech perception and vocabulary development. For example, several studies of pediatric CI recipients found that greater amounts of preimplant residual hearing were associated with better speech perception and language outcomes (Cowan et al, 1997; Nicholas and Geers, 2006; 2007). Likewise, Phan et al (2016) found that more preimplant residual hearing (i.e., lower unaided better-ear PTA; 0.5, 1 , and $2 \mathrm{kHz}$ ) was associated with better speech discrimination performance at two to four weeks post-CI stimulation for infants $(\mathrm{N}=$ 17) with a mean age of 16.4 months (standard deviation [SD = 3.6]). Notably, this result was found even though the average "best unaided" PTA was $108.7 \mathrm{~dB}$ HL and ranged from 87 to $120 \mathrm{~dB}$ HL. Thus, Phan et al (2016) concluded that even very-limited residual preimplant hearing may facilitate speech discrimination abilities in the time period immediately following implantation.

In contrast to the many studies that report degree of preimplant hearing for eventual pediatric CI recipients, there are few reports on the second factor affecting audibility, namely, HA fittings before CI surgery. Although some clinicians and researchers may report the age at which HA use was initiated (Geers et al, 2013; Davidson et al, 2014), very few report on the fittings of the HAs themselves or on the amount of speech that is audible during HA trials. For children with less profound degrees of HL, there are some studies of HA fittings. In studies of children with mild to severe HL, McCreery et al (2013) and McCreery et al (2015) report that HA fittings were variable. In these two studies, the proximity of the HA output to prescribed pediatric targets (Desired Sensation Level-[DSL]) was examined for each child's HAs (defined as "deviation from target"). Then, aided Speech Intelligibility Indices (SII; ANSI S3.5, 1997) were calculated and compared with published normative ranges for children (Bagatto et al, 2011; 2016; Moodie et al, 2017). Approximately $55 \%$ of the children's HAs deviated significantly from their prescribed targets in both ears (McCreery et al, 2013). "Deviations from target" tended in the direction of underfitting, such that children's HAs with greater deviations tended to have SIIs that were lower (poorer) than the normative range for a given degree of HL; $35 \%$ of the HAs had SIIs that were below the published normative range for their severity of $\mathrm{HL}$ (McCreery et al, 2015).

For children with profound levels of HL, however, there is only one report on HA fittings (Strauss and van Dijk, 2008) and none that report the amount of speech that is audible when aided (SII data). In several reports of an association between better preimplant residual hearing and better postimplant outcomes (e.g., Cowan et al, 1997; Nicholas and Geers, 2006; 2007), there is an assumption that speech was optimally audible during the preimplant period. Yet, these studies provided no verification or documentation of the aided HA fit (i.e., HA verification) and/or quantification of the audibility of speech. One study, by Davidson and Skinner (2006), examined relations among PTAs, SIIs, and speech recognition scores for 26 children with severe to profound HL. Although HA fits were not reported explicitly, SIIs for two input speech levels (soft: $55 \mathrm{~dB}$ SPL [at the time of this study, the lowest input level available on the Audioscan system was $55 \mathrm{~dB}$ SPL] and raised: $70 \mathrm{~dB}$ SPL) were calculated using the Audioscan Verifit System (Etymonic Design Inc., Ontario, Canada). The mean better-ear unaided PTA for the group was $79 \mathrm{~dB}$ HL (range: 60-98 dB HL), and mean SIIs were $27 \%$ and $42 \%$ for the soft and raised input levels, respectively. Although the calculated SIIs were relatively low, they were significantly correlated with speech recognition scores on the Lexical Neighborhood Test (LNT; Kirk et al, 1995) test at both levels. That is, soft-input SIIs were significantly correlated with speech recognition scores for soft speech ( $50 \mathrm{~dB}$ SPL) and raised-input SIIs were significantly correlated with speech recognition scores for loud speech (70 dB SPL).

Because many children with profound HL will eventually receive one or two CIs, some may question the value of ensuring good, or best-possible, HA fits and speech audibility during the period when these children 
are using HAs. Before implant surgery, the everyday audibility of speech will depend, as stated previously, on both a child's degree of HL (e.g., preimplant unaided PTA) and on how well his/her HA is fit. Clinically, although the audiologist has no influence on a child's degree of HL (i.e., unaided PTA), the audiologist does have some influence on how well fit a child's HAs are such that speech is as audible as possible. Presumably, any improvement in the audibility of speech, albeit even small increases in aided SII, might help the child while likely not causing any harm. Thus, a careful and systematic examination of HA fittings is warranted. In addition, because children with greater degrees of residual hearing are now potential CI candidates, the need for careful HA fitting and adjustment, and documentation thereof, are critical (Gifford et al, 2010; Sampaio et al, 2011; Mowry et al, 2012). Such information may assist in determining CI candidacy and with decisions about continued use of an HA at a nonimplanted ear (i.e., bimodal use). Thus, the primary aim of this project is to characterize HA fittings and aided audibility of speech for pediatric HA users with severe to profound $\mathrm{HL}$ who later received $\mathrm{CI}(\mathrm{s})$. A secondary aim is to examine the relation between preimplant aided audibility and postimplant speech perception.

\section{METHODS}

\section{Participants}

Participants were drawn from a larger study examining the effects of early acoustic hearing for pediatric CI recipients, and were recruited from audiology centers and oral schools for the deaf across the United States. Participants met the following criteria: severe to profound sensorineural HL in at least the poorer ear (a few exceptions were children with etiologies of auditory neuropathy spectrum disorder, enlarged vestibular aqueduct, and/or cytomegalovirus), HL that was congenital or acquired before 15 months of age, educated in an oral communication setting, and at least one CI surgery before 4.5 years of age. For this present study (HA fits \& SII), data were collected retrospectively using pre-CI audiologic records.

Although there are 117 children in the larger study, only those participants with HA verification information in their pre-CI audiological records were included in this present study. In addition, for some participants, more than one ear fit the inclusion criteria (e.g., child has bilateral CIs and HA verification data for both ears). When available, data collected from the participants' records included gender, age at $\mathrm{HA}$ fitting(s), age at $\mathrm{CI}(\mathrm{s})$, unaided air conduction thresholds, aided thresholds, etiology of HL, device history (i.e., type of device, manufacturer, and date fit), present device use (i.e., simultaneous bilateral CIs, sequential bilateral CIs, and bimodal devices), and manufacturer of devices (HA manufacturers: Phonak, Widex, Oticon, and Unitron; CI manufacturers: Cochlear Americas [Englewood, CO], Advanced Bionics [Valencia, CA], Med-EL [Innsbruck, Austria]). A total of 45 pediatric participants ( 23 female and 22 male) consisting of 71 ears were included in this present study (see Table 1). These participants include bilateral CI users, simultaneous $(\mathrm{n}=9)$ and sequentially $(\mathrm{n}=21)$ implanted, and bimodal CI users $(n=15)$, that is, those who use an $\mathrm{HA}$ at the nonimplanted ear. For these 45 participants, the average age at the time of the test (testing performed for the larger study) was 6.8 years $(\mathrm{SD}=1.3)$, and the average age at first CI was 2.3 years $(\mathrm{SD}=1.1)$ with a mean preimplant unaided PTA of $99 \mathrm{~dB} \mathrm{HL}(\mathrm{SD}=16)$. For the other 72 participants from the larger study, that is, those who did not have preimplant HA-fitting data available in their audiological records, the average age at the time of test was 7.2 years $(\mathrm{SD}=1.3)$, average age at first CI was 2.0 years $(\mathrm{SD}=1.1)$, and mean preimplant unaided PTA was $98 \mathrm{~dB}$ HL $(\mathrm{SD}=17)$.

\section{HA Verification}

HA-fitting data from Audioscan Verifit ${ }^{\text {TM }}$ records were collected and included the following (when available): measured HA output in dB SPL for running speech (carrot passage) at different input levels ranging from soft to loud and Audioscan softwarecomputed SIIs (ANSI S3.5, 1997) for different input levels (when available). For most participants, agerelated average real ear to coupler difference values were used to simulate in situ measurements of HA output in the coupler of the Audioscan system. From audiology records and HA-fitting reports, participants who used HAs with frequency-lowering capability,

Table 1. Participants and Ears with Verification Data; HAs with Active Frequency Lowering

\begin{tabular}{lccccc}
\hline & \multicolumn{3}{c}{ Number of Participants } & & Number of \\
\cline { 2 - 3 } HA Verification Data & $\begin{array}{c}\text { With Data for } \\
\text { One Ear }\end{array}$ & $\begin{array}{c}\text { With Data for } \\
\text { Two Ears }\end{array}$ & Total & Aided Ears & $\begin{array}{c}\text { Number of HAs with Active } \\
\text { Frequency Lowering }\end{array}$ \\
\hline Any input level & 19 & 26 & 45 & 71 & 35 \\
$\quad$ Soft level & 16 & 14 & 30 & 44 & 26 \\
Conversational level & 16 & 20 & 36 & 56 & 30 \\
Loud level & 13 & 17 & 30 & 47 & 23 \\
\hline
\end{tabular}

Note: Number of participants and aided ears with available HA verification data, and number of HAs with active frequency lowering, in total (any input level) and for three verification speech input levels. 
specifically nonlinear frequency compression, were identified. Centers differed in their definition of input levels, with $5 \mathrm{~dB}$ differences noted at each level: soft $=50$ or $55 \mathrm{~dB}$ SPL, conversational $=60$ or $65 \mathrm{~dB}$ SPL, and loud $=70$ or 75 dB SPL. From Audioscan Verifit ${ }^{\text {TM }}$ records, deviations of HA output from DSL targets (defined as HA output minus DSL target) were examined for octave frequencies from 250 to $4000 \mathrm{~Hz}$, when available. Only the deviations for the conversational speech input level ( 60 or $65 \mathrm{~dB}$ SPL) are reported here $(\mathrm{N}=56 \mathrm{HAs} ; 56$ ears) because that level was most frequently used with the Verifit $^{\text {TM }}$ (see Table 1). In some audiologic records, graphical data were provided in lieu of numerical data; in these instances, a MATLAB (Mathwords, Natick, MA) script named Graph Picker was used to estimate the numerical values of prescriptive targets and HA output at each frequency. Finally, aided SIIs as a function of unaided preimplant PTA for these participants' ears were compared with normative data from the University of Western Ontario Pediatric Audiological Monitoring Protocol (UWO-PedAMP) (Bagatto et al, 2011; 2016; Moodie et al, 2017).

\section{Speech Recognition Performance}

As part of the larger study, participants, at ages five to eight years, were administered the LNT while using their bilateral devices ( $2 \mathrm{CIs}$ or $\mathrm{CI}+\mathrm{HA}$ ). This open-set word recognition test consists of monosyllabic, 50-word lists drawn from the vocabulary of three- to five-year-old typically developing children. Each child heard one 50-word list in the presence of four-talker babble presented with an 8-dB signal to noise ratio, with the speech level at 60 $\mathrm{dB}(\mathrm{A})$. The speech tests were administered in a sound booth, with the prerecorded signals presented via an audiometer to a speaker located at $0^{\circ}$ azimuth about 3 feet from the child. The child was instructed to repeat what he/she heard. A percent-correct word score was computed that represents the percent of words (responses) that were recognizable as the target word.

\section{STATISTICAL ANALYSES}

$\mathrm{T}$ 1 o determine whether the deviations from target (HA output - DSL target) were significantly greater than zero, 95\% confidence intervals of the mean deviation (upper and lower limits [LL, UL]) for each frequency $(250,500,1000,2000$, and $4000 \mathrm{~Hz})$ were calculated. In addition, $t$-tests were used to compare preimplant-aided SIIs of HAs of listeners in each of the three present device groups (simultaneous CIs, sequential CIs, and bimodal) and to compare aided SIIs across ears for two groups of listeners: those who were sequentially implanted with CIs and those who used bimodal devices. Correlational analyses were used to examine the relationship between preimplant-aided SIIs (for the ear receiving the first CI) and postimplant speech perception in noise. In addition, correlational analyses were used to explore relationships between preimplant-aided SIIs and demographic data.

\section{RESULTS}

$\mathrm{F}$ or the ears in this study, that is, those with HA verification data, mean unaided preimplant thresholds at octave frequencies from 250 to $4000 \mathrm{~Hz}$ are shown in Figure 1. Mean preimplant thresholds are severe to profound at all octave frequencies.

\section{Deviation from (Prescriptive DSL) Target}

Deviations from prescriptive target, defined as "HA output minus DSL target," at octave frequencies from 250 to $4000 \mathrm{~Hz}$ for conversational speech input levels ( 60 or 65 dB SPL) are shown in Figure 2. Because HA outputs were not available at every frequency for every ear, there are different $\mathrm{Ns}$ (different numbers of ears) at each frequency. Table 2 shows, for each octave frequency, the mean, SD, and LL and UL of the $95 \%$ confidence interval of the mean for the deviations from prescriptive target for 56 HAs (or, equivalently, for 56 ears). Overall, for conversational speech levels, HA outputs were very close to prescriptive targets, that is, the deviations from target are close to zero. At $4000 \mathrm{~Hz}$, however, the average HA output was significantly below the prescriptive (DSL) target, with a mean deviation of -14.2 $\mathrm{dB}$. At all other frequencies, deviations from target were not significantly different from zero. Because this analysis included different numbers of HAs (ears) at each frequency, the analysis was repeated using only those HAs

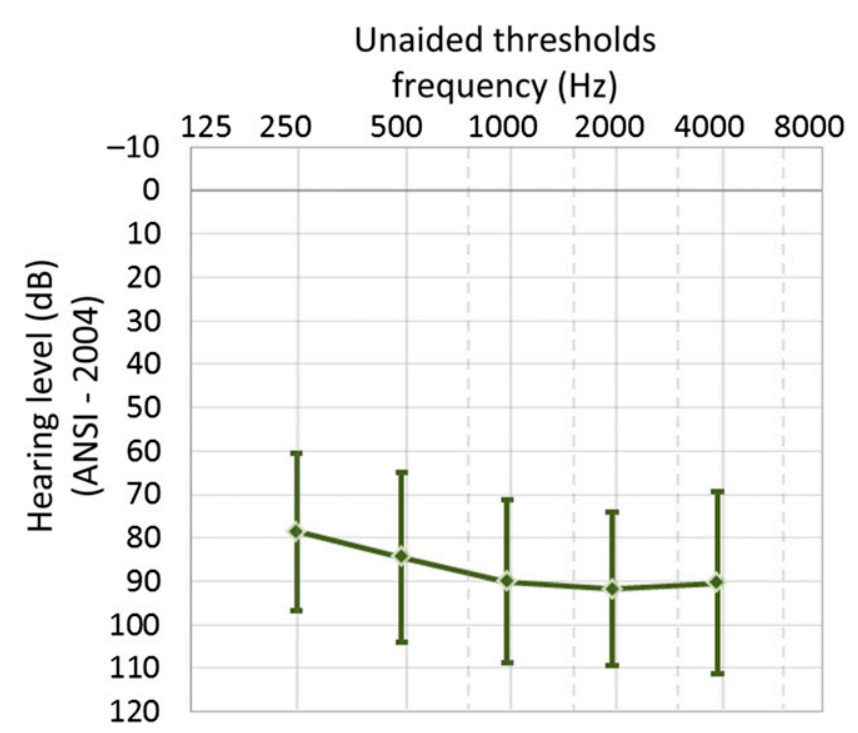

Figure 1. Mean unaided preimplant thresholds are plotted in $\mathrm{dB}$ HL with +1/-1 SD at octave frequencies from 250 to $4000 \mathrm{~Hz}$, for the pediatric participants in this study $(\mathrm{N}=45)$. The mean unaided thresholds are $78,83,89,91$, and 88 , at octave frequencies $250,500,1000,2000$, and $4000 \mathrm{~Hz}$, respectively. (This figure appears in color in the online version of this article.) 


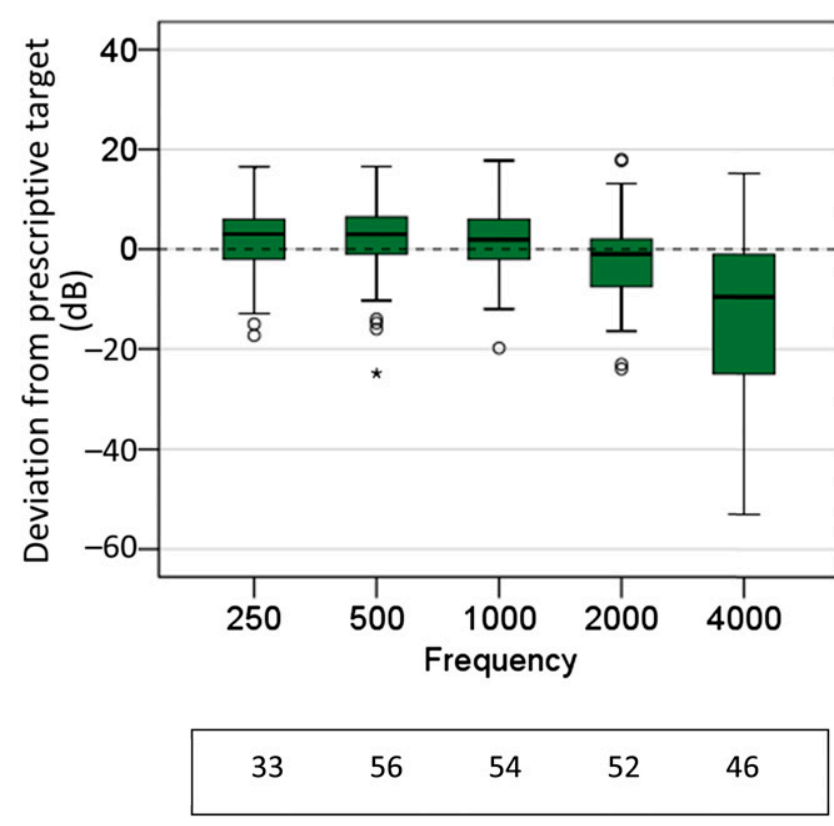

Figure 2. Box plots of "deviation from prescriptive target" for conversational input levels (60 or $65 \mathrm{~dB}$ SPL); box plots include HAs with active frequency lowering, from a total of 56 HAs. In each box plot, median and interquartile range (IQR) are indicated by the thick line in the middle, and the distance between the upper and lower extent of the box. Whiskers indicate the minimum and maximum values, except for outliers (circles indicate outliers $>1.5$ IQR's but $<3$ IQR's from the median, whereas asterisks represent outliers $>3$ IQR's from the median). Numbers of HAs included in the box plots are listed below the graph at each octave frequency. The activation of frequency lowering in some HAs (30 out of 56) is reflected in larger deviations from prescriptive target at the high frequencies, particularly $4000 \mathrm{~Hz}$. Use of frequency lowering is presumably related to poor audibility at high frequencies for some participants and suggests that clinicians were likely seeking to improve high-frequency audibility when it was not achieved with conventional amplification. (This figure appears in color in the online version of this article.)

(participants' ears) for which conversational-level data were available at all octave frequencies $(\mathrm{N}=28)$. The pattern of results remained unchanged, namely, only at 4000 $\mathrm{Hz}$ did deviations from target differ significantly from 0 (at $4000 \mathrm{~Hz}$, mean deviation $=-17 \mathrm{~dB})$.

Figure 3 and Table 3 show analogous results, deviations from prescriptive DSL target, but excluding HAs using fre- quency compression. Again, deviations from target are shown and provided at octave frequencies from 250 to $4000 \mathrm{~Hz}$ for conversational speech input levels (60 or $65 \mathrm{~dB}$ SPL), and again, because HA outputs were not available at every frequency for every ear, there are different $\mathrm{Ns}$ at each frequency. At $1000 \mathrm{~Hz}$, the average deviation was $3 \mathrm{~dB}(\mathrm{SD}=7.2)$ above target and was significantly different from zero. At all other frequencies, the deviations from target were not significantly different from zero.

\section{SII}

Audioscan software-computed aided SIIs for soft, conversational, and loud speech input levels are shown, using box plots, in Figure $4(\mathrm{~N}=52$ aided ears; only those aided ears with SII values at all three speech-input levels are shown in this figure). As expected, SIIs are lowest for soft levels (mean $=22 ; \mathrm{SD}=19)$, slightly higher for conversational levels (mean $=30 ; \mathrm{SD}=20$ ), and then, again slightly higher for loud levels ( mean $=36$; $\mathrm{SD}=20$ ). SIIs were also examined for various subgroups of participants. For the simultaneously implanted bilateral CI participants $(\mathrm{N}=9)$, the aided SII associated with the better ear's preimplant HA records are used. For the sequentially implanted bilateral CI participants $(\mathrm{N}=21)$, the aided SII associated with the first-CI ear's preimplant HA records are used, and for the participants with bimodal devices $(\mathrm{N}=15)$, the aided SII associated with the (only) CI ear's preimplant HA records are used. For aided ears of listeners in these present "device" groups, mean preimplant SII values for conversational speech input levels were $35(\mathrm{SD}=24)$ for the simultaneous bilateral CI group, $27(\mathrm{SD}=19)$ for the sequential bilateral $\mathrm{CI}$ group, and $30(\mathrm{SD}=22)$ for the bimodal group. Mean SII was not significantly different across these three groups. Finally, SIIs for conversational speech input levels were also compared, across ears, for the participants in the sequential CI and bimodal device groups. (A similar comparison was not done for participants who received bilateral CIs simultaneously because presumably, they would have had similar unaided preimplant PTAs in both ears.) For the sequential bilateral CI participants, preimplant-aided SIIs for the first CI

Table 2. Deviations from Target

\begin{tabular}{|c|c|c|c|c|}
\hline Frequency $(\mathrm{Hz})$ & $N$ & Mean $(\mathrm{dB})$ & $\mathrm{SD}(\mathrm{dB})$ & $\begin{array}{c}\text { 95\% Confidence } \\
\text { Interval }[\mathrm{LL}, \mathrm{UL}](\mathrm{dB})\end{array}$ \\
\hline$\overline{250}$ & 33 & 1.4 & 7.8 & {$[-1.4,4.1]$} \\
\hline 500 & 56 & 2.0 & 7.8 & {$[-0.1,4.1]$} \\
\hline 1000 & 54 & 1.5 & 7.2 & {$[-0.5,3.5]$} \\
\hline 2000 & 52 & -2.1 & 8.6 & {$[-4.5,0.3]$} \\
\hline 4000 & 46 & -14.2 & 17.3 & {$[-19.3,-9.1]^{\star}$} \\
\hline
\end{tabular}

*Denotes significant.

Notes: Deviations from (prescriptive DSL) target ("HA output minus target") for conversational speech input levels and for all HAs. The number of ears (N), mean, SD, and the LL and UL of the 95\% confidence intervals of the mean are listed. If the $95 \%$ confidence interval does not include zero, then the mean deviation from prescriptive target is considered significantly different from zero; any such instances are indicated with an asterisk. 


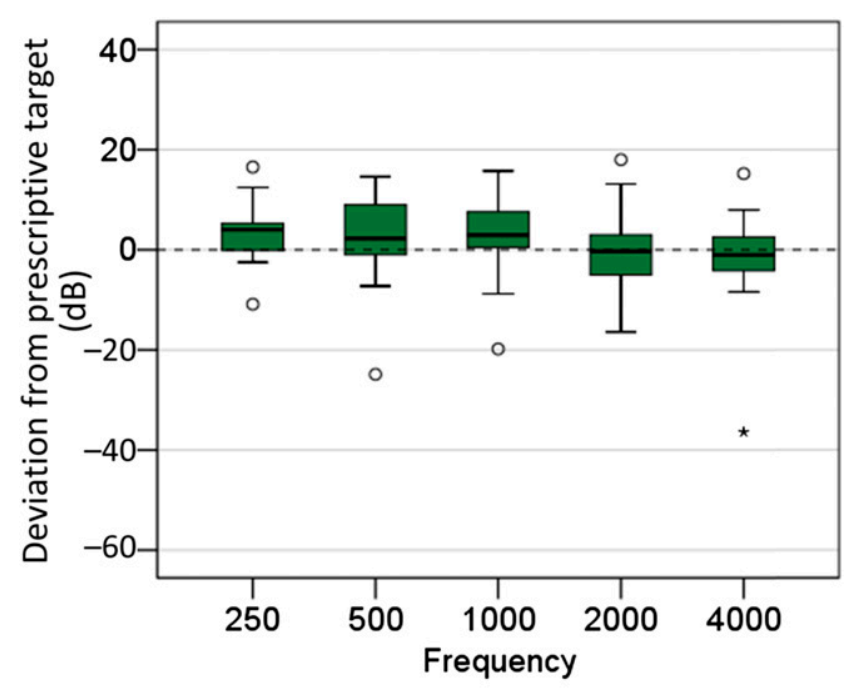

$\begin{array}{lllll}11 & 26 & 26 & 26 & 23\end{array}$

Figure 3. Box plots of "deviation from prescriptive target" for conversational input levels ( 60 or $65 \mathrm{~dB}$ SPL); box plots exclude HAs with active frequency lowering, from a total of 26 HAs. For details on the box plots, see the caption for Figure 2. Numbers of HAs included in the box plots are listed below the graph at each octave frequency. (This figure appears in color in the online version of this article.)

ear $($ mean $=28 ; \mathrm{SD}=28)$ were generally lower than preimplant-aided SIIs for the second CI ear (mean $=37$; $\mathrm{SD}=21$ ), although this difference was not statistically significant. For the bimodal device users, aided SIIs for the preimplant ears (mean $=30$; $\mathrm{SD}=22$ ) were significantly lower $\left[t_{(14)}=-3.34, p<0.01\right]$ than the aided SIIs for the ears currently wearing HAs (mean $=52 ; \mathrm{SD}=18$ ).

In Figure 5, Audioscan software-computed aided SIIs, for these participants' ears for conversational speech input levels, are plotted as a function of unaided, preimplant PTA. Also shown are the normative data from the UWO-PedAMP (Bagatto et al, 2011; 2016; Moodie et al, 2017). Shown in red is the line of best fit for the 67 ears in our sample; these are all the aided ears with SIIs available for a conversational-level speech input. (For four aided ears, SIIs were available only for soft- and/or loud-level speech inputs.) The red line departs from the UWO-PedAMP data, especially for unaided PTAs $>75 \mathrm{~dB}$ HL. Note that there are a few ears with PTAs in the moderate loss range; these ears belong to children who had a diagnosis of progressive HL (i.e., enlarged vestibular aqueduct or cytomegalovirus) or auditory neuropathy spectrum disorder.

\section{Postimplant Speech Recognition Scores}

The mean score for the LNT word list presented in noise $(+8 \mathrm{~dB}$ signal to noise ratio) was $60 \%$ correct (SD: 22.7; range 0-92\%).

\section{Correlations}

Aided SIIs (at conversational speech input levels) were not significantly correlated with age at first HA nor were they correlated with age at first CI. As expected, unaided PTA was significantly correlated with preimplant-aided conversational-level SIIs $(r=$ $-0.936, p<0.01$ ) (see Figure 5). Preimplant-aided conversational SIIs for the first CI ear were also significantly correlated with postimplant LNT in noise scores $(r=0.35, p<0.05)$, when tested in these same children at ages five to eight years. Interestingly, however, age at first CI was not correlated with postimplant LNT in noise scores.

\section{DISCUSSION}

$T$ 1 he pediatric CI candidacy process typically includes a trial period with an HA, or HAs, before any possible CI surgery. Not only does this practice assist in determining CI candidacy but it also allows the child to receive some audibility of speech, although perhaps quite limited. Pediatric Amplification Guidelines (AAA, 2013) provide specific protocols for achieving and verifying audibility targets with HAs. Studies from children with mild to severe loss have demonstrated

Table 3. Deviations from Target Excluding Frequency Lowering

\begin{tabular}{lcccc}
\hline Frequency $(\mathrm{Hz})$ & $\mathrm{N}$ & Mean $(\mathrm{dB})$ & $\mathrm{SD}(\mathrm{dB})$ & $\begin{array}{c}\text { 95\% Confidence } \\
\text { Interval }[\mathrm{LL}, \mathrm{UL}](\mathrm{dB})\end{array}$ \\
\hline 250 & 11 & 3.3 & 7.3 & {$[-1.6,8.2]$} \\
500 & 26 & 2.7 & 8.0 & {$[-0.5,5.9]$} \\
1000 & 26 & 3.0 & 7.2 & {$[0.1,5.9]^{*}$} \\
2000 & 26 & -0.7 & 9.0 & {$[-3.9,2.6]$} \\
4000 & 23 & -1.6 & 9.4 & {$[-5.7,2.4]$} \\
\hline
\end{tabular}

*Denotes significance.

Notes: Deviations from (prescriptive DSL) target ("HA output minus target") for conversational speech input levels, excluding HAs that use frequency compression. The number of ears (N), mean, SD, and the LL and UL of the $95 \%$ confidence intervals of the mean are listed. If the $95 \%$ confidence interval does not include zero, then the mean deviation from prescriptive target is considered significantly different from zero; any such instances are indicated with an asterisk. 


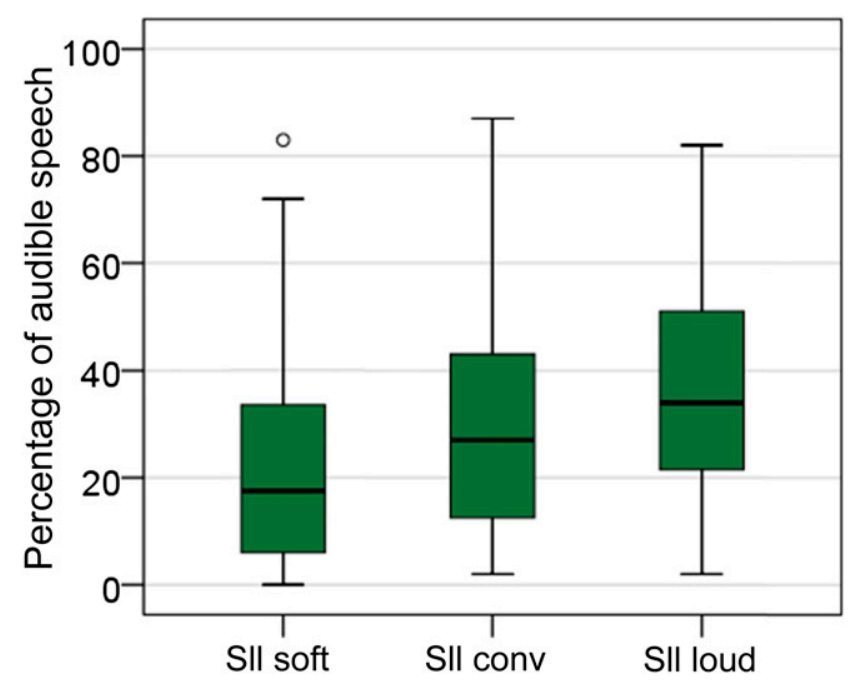

Figure 4. Box plots of aided SIIs (percentage of audible speech), computed from Audioscan software, for three speech input levels. Only those aided ears with SII values at all three speech-input levels are shown in this figure $(\mathrm{N}=52)$. (This figure appears in color in the online version of this article.)

positive effects of well-fit HAs on spoken language outcomes (McCreery et al, 2015). Thus, for pediatric recipients, it is reasonable to attribute the positive effects of preimplant residual hearing on post-CI outcomes inpart to the audibility of speech provided by HAs.

This study sought to characterize, for pediatric CI recipients, preimplant HA fittings and aided audibility (which is, of course, affected by PTAs and HA fittings). Of a total of 117 participants in a larger study $(\mathrm{NIH}$

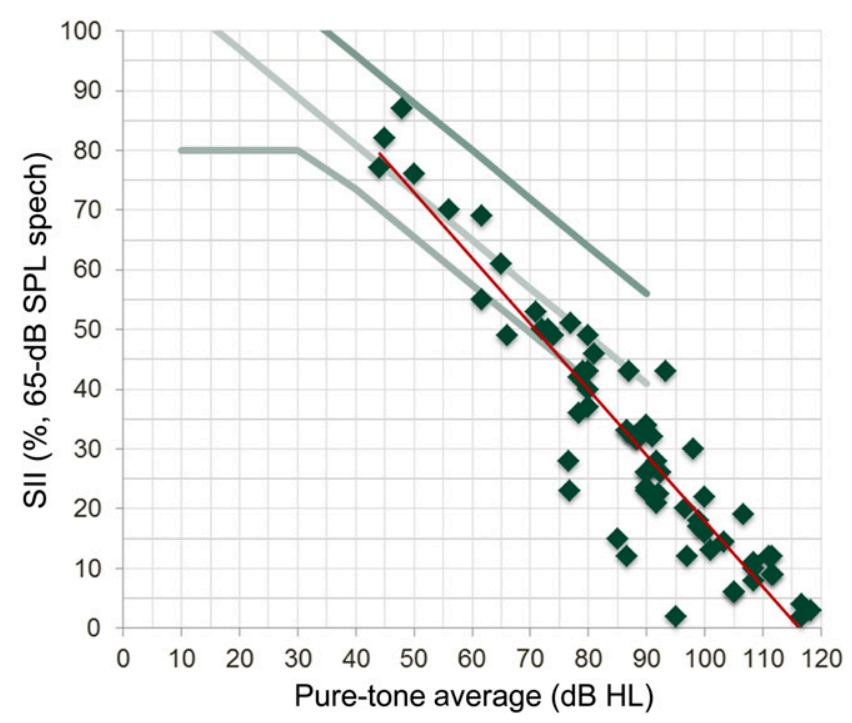

Figure 5. Aided SII vs. unaided preimplant PTA for these participants' ears $(\mathrm{N}=67)$ are shown individually by diamonds, and the line of best fit is shown in red. Normative data are from the UWOPedAMP. For the normative data, the light gray line represents average aided SII values and the dark gray lines represent SDs (2 SD above and $1 \mathrm{SD}$ below) about the mean normative data. (This figure appears in color in the online version of this article.)
R01 DC012778), preimplant HA fitting records were retrievable for 45 participants (approximately $38 \%$ ). Many $\mathrm{CI}$ and audiology centers reported that such data were not routinely saved in their patient records. Although there was some variation in the speech input levels (soft, conversational, or loud) used for matching prescriptive targets, most centers used a 60-65-dB SPL level to represent average conversational speech. Overall, in this sample of children with severe to profound HLs, who later received at least one CI, HAs were generally closely matched to prescriptive targets. Because HA fitting data were unavailable for most of the participants (i.e., the other $62 \%$ ), it is uncertain whether these data are representative of pediatric CI recipients in general. These 45 participants, however, are seemingly no different from the remaining 72 participants with respect to preimplant PTA, age at first CI, and age at the time of speech perception tests (see description of participants in "Methods").

For children with mild to profound losses, studies evaluating proximity to HA fitting targets have found that a substantial number of children have HA outputs that deviate significantly from target (Strauss and van Dijk, 2008; McCreery et al, 2013; Ching et al, 2015; McCreery et al, 2015). Moreover, some researchers suggest that deviations from target increase with more severe HLs, especially deviations at high frequencies such as $4-6 \mathrm{kHz}$ (Ching et al, 2015). Yet, for speech recognition and ultimate development of spoken language skills, high-frequency audibility is critical for children (Stelmachowicz et al, 2004; Stelmachowicz et al, 2008). Thus, clinicians do their best, using any method possible-including frequency-lowering methods-to provide at least limited high-frequency audibility before receiving CIs. For the present study, children with HA outputs that deviated significantly from target at 4000 $\mathrm{Hz}$ were those who used HAs with active frequency lowering. This type of processing shifts (or lowers) highfrequency information to lower frequency regions, where listeners generally have better residual hearing (Simpson et al, 2005; Glista et al, 2009). This type of processing aims overcoming limitations in high-frequency audibility resulting from a combination of poor residual hearing and limited HA bandwidths. When the data in this present study were analyzed with frequency-lowering HAs excluded, deviations from target were not significantly different from zero. This result makes sense because HAs with frequency-lowering do not amplify high-frequency energy (beyond a specified cut-off frequency), but instead transmit high-frequency information at lower frequencies.

The aided SIIs, for these participants, estimate the audibility of speech, preimplant, in specific circumstances. When the distance between a talker and a child was approximately $1 \mathrm{~m}$, then approximately $22 \%$ of softly spoken speech would have been audible (mean preimplant SII $=22$ ). Similarly, $\sim 30 \%$ and $\sim 36 \%$ of conversationally 
spoken and loudly produced speech would have been audible, respectively, to an infant (preimplant) at this same 1-m distance. All these values are very low. Stiles et al (2012) suggest that for children with mild to moderately severe HL, those with aided SII values $<65$ exhibit greater delays in vocabulary development. For the children in the present study, with more severe to profound HL, these low SIIs represent inadequate audibility of speech (even of "loud" speech) such that CI surgery appears clearly warranted for all participants (recall, there was no significant difference in preimplant-aided SII across the three device groups). For the children who currently use bimodal devices, the significant difference in aided SII of the preimplant ear versus that of the present HA ear indicates that the ear with poorer hearing was chosen for implantation.

Calculation of the aided SII depends on both unaided PTA and on the fit of the HA to DSL target (McCreery et al, 2015). Consequently, it is unsurprising that for this study, SII and unaided PTA are highly correlated with each other, a result consistent with other studies (Sininger et al, 2010; McCreery et al, 2015). The correlation $(r=-0.936, p<0.01)$ is especially strong in this study because HA fits were all very close to DSL target. That is, overall, deviations from target were approximately zero. The exact relation, between PTA and SII, for children with HL that range from mild to severe, is shown by the UWO-PedAMP normative data (Bagatto et al, 2011; 2016; Moodie et al, 2017). The regression line for SII versus PTA in the present sample, however, departs from the normative data for PTAs $>75 \mathrm{~dB}$ HL. This seems likely due to the fact that the normative values were obtained from children with greater residual hearing (i.e., with less severe HL) than those in the present sample. Despite these reasonably well-fit HAs, these children were deemed ultimately and quite appropriately as candidates for CIs.

Interestingly, despite limited residual hearing (PTA) and limited aided audibility (SII), there was a positive and significant, albeit somewhat weak, correlation between aided SII and post-CI speech perception in noise (and, equivalently, between unaided PTA and post-CI speech perception in noise). These results along with those from other studies support the notion that even limited audibility before receiving a CI may be beneficial for speech recognition (Cowan et al, 1997; Nicholas and Geers, 2006; 2007; Sininger et al, 2010). Unlike other studies (Dowell et al, 2002; Geers et al, 2013), age at first CI was not correlated with speech perception in noise for this sample. The potential effects of age at first CI on eventual speech perception ability may have been mitigated by the consistent use of HAs, before receiving CIs, for the participants in this study. Regardless, this weak positive correlation provides further support that early audibility of speech, presumably provided via HAs, could have positive effects for children who subsequently receive CIs.
There are several considerations that may limit generalizing the results of the present study to other pediatric CI recipients. Although most of the participants in the present study had HAs that were fit to prescriptive target, these participants represent only about onethird of the total population of participants in the larger study. It is possible that centers that keep carefully documented HA-fitting records may be more likely to place a high priority on preimplant fitting procedures and thus bias the results toward more optimal HA fittings. In addition, all of the preimplant data were obtained retrospectively from centers across the United States several years after the measurements were obtained. Thus, differences in equipment, procedures, and personnel, across centers and across time, may contribute to variations in these measurements. There are, of course, many demographic and child factors that are very likely to affect post-CI outcomes (i.e., maternal education, enrollment in early intervention programs, etc.). The sample size $(\mathrm{N}=71 \mathrm{ears})$ in the present study, however, is not adequate for sophisticated regression analyses that would allow quantitative determination of the contributions of child, family, educational, and audiological variables to post-CI outcomes. Rather, the primary scope of the present study was to characterize preimplant-aided audibility profiles of children who receive CIs and determine whether preimplant audibility, as measured by HA fitting parameters, is related to postimplant outcomes. An examination of aided audibility (SII), using the HA fit to target, may guide clinicians in determining CI candidacy and should be encouraged. As children with more residual hearing are considered for CIs, such an examination may be more critically important than in the past.

Acknowledgments. We thank the following centers for their participation in this study: Central Institute for the Deaf, St. Louis, MO; St. Louis Children's Hospital, St. Louis, MO; Moog Center for Deaf Education, St. Louis, MO; Memphis Oral School for the Deaf, Germantown, TN; Hearts for Hearing, Oklahoma City, OK; Child's Voice, Chicago, IL; Weingarten Children's Center, Redwood City, CA; University of Texas-Dallas, Dallas, TX; CCHAT Center (Children's Choice for Hearing and Talking), Sacramento, CA; PEI (Presbyterian Ear Institute), Albuquerque, NM; Children's Hospital of Philadelphia, Philadelphia, PA; University of Minnesota Masonic Children's Hospital, Minneapolis, MN; Arkansas Children's Hospital, Little Rock, AR; Ohio Valley Voices, Loveland, $\mathrm{OH}$; Listen and Talk, Seattle, WA; Vanderbilt University, Nashville, TN; Barton G Kids Hear Now Cochlear Implant Family Resource Center, University of Miami, Miami, FL.

\section{REFERENCES}

American Academy of Audiology (AAA). (2013) American Academy of Audiology Clinical Practice Guidelines: Pediatric Amplification. Reston, VA: American Academy of Audiology. 
American National Standards Institute (ANSI). (1997) Methods for Calculation of the Speech Intelligibility Index (ANSI S3.5). New York, NY: ANSI.

Bagatto M, Moodie S, Brown C, Malandrino A, Richert F, Clench D, Scollie S. (2016) Prescribing and verifying hearing aids applying the American academy of audiology pediatric amplification guideline: protocols and outcomes from the Ontario infant hearing program. J Am Acad Audiol 27(3):188-203.

Bagatto MP, Moodie ST, Malandrino AC, Richert FM, Clench DA, Scollie SD. (2011) The University of Western Ontario Pediatric Audiological Monitoring Protocol (UWO PedAMP). Trends Amplif 15(1):57-76.

Ching TY, Quar TK, Johnson EE, Newall P, Sharma M. (2015) Comparing NAL-NL1 and DSL v5 in hearing aids fit to children with severe or profound hearing loss: goodness of fit-to-targets, impacts on predicted loudness and speech intelligibility. J Am Acad Audiol 26(3):260-274.

Cowan RSC, DelDot J, Barker EJ, Sarant JZ, Pegg P, Dettman S, Galvin KL, Rance G, Hollow R, Dowell RC, Pyman B, Gibson WP, Clark GM. (1997) Speech perception results for children with implants with different levels of preoperative residual hearing. Am $J$ Otolaryngol 18(6, Suppl):S125-S126.

Davidson LS, Geers AE, Nicholas JG. (2014) The effects of audibility and novel word learning ability on vocabulary level in children with cochlear implants. Cochlear Implants Int 15(4):211-221.

Davidson LS, Skinner MW. (2006) Audibility and speech perception of children using wide dynamic range compression hearing aids. Am J Audiol 15(2):141-153.

Dowell RC, Dettman SJ, Blamey PJ, Barker EJ, Clark GM. (2002) Speech perception in children using cochlear implants: prediction of long-term outcomes. Cochlear Implants Int 3(1):1-18.

Geers A, Davidson L, Uchanski R, Nicholas J. (2013) Interdependence of linguistic and indexical speech perception skills in schoolaged children with early cochlear implantation. Ear Hear 34(5): $562-574$

Geers AE, Moog JS, Biedenstein J, Brenner C, Hayes H. (2009) Spoken language scores of children using cochlear implants compared to hearing age-mates at school entry. J Deaf Stud Deaf Educ 14(3):371-385.

Gifford RH, Dorman MF, Shallop JK, Sydlowski SA. (2010) Evidence for the expansion of adult cochlear implant candidacy. Ear Hear 31(2):186-194.

Glista D, Scollie S, Bagatto M, Seewald R, Parsa V, Johnson A. (2009) Evaluation of nonlinear frequency compression: clinical outcomes. Int J Audiol 48(1):632-644.

Kirk KI, Pisoni DB, Osberger MJ. (1995) Lexical effects on spoken word recognition by pediatric cochlear implant users. Ear Hear 16(5):470-481.

McCreery RW, Bentler RA, Roush PA. (2013) The characteristics of hearing aid fittings in infants and young children. Ear Hear 34(6):701-710.

McCreery RW, Walker EA, Spratford M, Bentler R, Holte L, Roush P, Oleson J, Buren JV, Moeller MP. (2015) Longitudinal predictors of aided speech audibility in infants and children. Ear Hear 36(1, Suppl):24S-37S.
Moodie STF, Scollie SD, Bagatto MP, Keene K, The Network of Pediatric Audiologists of Canada. Fit-to-targets for the desired sensation level version 5.0a hearing aid prescription method for children. Am J Audiol 26(3):251-258.

Mowry SE, Woodson E, Gantz BJ. (2012) New frontiers in cochlear implantation: acoustic plus electric hearing, hearing preservation, and more. Otolaryngol Clin North Am 45(1):187-203.

Nicholas JG. (2000) Age differences in the use of informative/ heuristic communicative functions in young children with and without hearing loss who are learning spoken language. $J$ Speech Lang Hear Res 43:380-394.

Nicholas JG, Geers AE. (2006) Effects of early auditory experience on the spoken language of deaf children at 3 years of age. Ear Hear 27(3):286-298.

Nicholas JG, Geers AE. (2007) Will they catch up? The role of age at cochlear implantation in the spoken language development of children with severe to profound hearing loss. J Speech Lang Hear Res 50(4):1048-1062.

Phan J, Houston DM, Ruffin C, Ting J, Holt RF. (2016) Factors affecting speech discrimination in children with cochlear implants: evidence from early-implanted infants. J Am Acad Audiol 27(6):480-488.

Pittman AL, Schuett BC. (2013) Effects of semantic and acoustic context on nonword detection in children with hearing loss. Ear Hear 34(2):213-220.

Sampaio ALL, Araújo MFS, Oliveira CA. (2011) New criteria of indication and selection of patients to cochlear implant. Int J Otolaryngol 2011:573968.

Simpson A, Hersbach AA, McDermott HJ. (2005) Improvements in speech perception with an experimental nonlinear frequency compression hearing device. Int J Audiol 44(5):281-292.

Sininger YS, Grimes A, Christensen E. (2010) Auditory development in early amplified children: factors influencing auditorybased communication outcomes in children with hearing loss. Ear Hear 31(2):166-185.

Stelmachowicz PG, Nishi K, Choi S, Lewis DE, Hoover BM, Dierking D, Lotto A. (2008) Effects of stimulus bandwidth on the imitation of English fricatives by normal-hearing children. $J$ Speech Lang Hear Res 51(5):1369-1380.

Stelmachowicz PG, Pittman AL, Hoover BM, Lewis DE, Moeller MP. (2004) The importance of high-frequency audibility in the speech and language development of children with hearing loss. Arch Otolaryngol Head Neck Surg 130(5):556-562.

Stiles DJ, Bentler RA, McGregor KK. (2012) The Speech Intelligibility Index and the pure-tone average as predictors of lexical ability in children fit with hearing aids. J Speech Lang Hear Res 55(3): 764-778.

Strauss S, van Dijk C. (2008) Hearing instrument fittings of preschool children: do we meet the prescription goals? Int $J$ Audiol 47(1, Suppl):S62-S71.

Tomblin JB, Oleson JJ, Ambrose SE, Walker E, Moeller MP. (2014) The influence of hearing aids on the speech and language development of children with hearing loss. JAMA Otolaryngol Head Neck Surg 140(5):403-409. 